\title{
INFINITE PRODUCTS OF ANALYTIC MATRICES
}

\section{GEORGE D. BIRKHOFF}

In a large part of the theory of functions of a single complex variable the matrix of analytic functions rather than the single analytic function must be taken as the fundamental element. This is certainly the case for the functions defined by linear difference and differential equations.

The goal of the present paper is to show that the classical results of Weierstrass and Mittag-Leffler, treating of the formation of infinite products of functions with assigned singularities, admits of a natural extension to infinite products of matrices. The matrices considered will be square matrices of $n^{2}$ elements and of determinant not identically zero.

The concept of equivalence, which I have developed elsewhere, $\dagger$ lies at the basis of this extension: Let

$$
A(x)=\left[a_{i j}(x)\right] \text { and } B(x)=\left[b_{i j}(x)\right] \quad(i, j=1, \cdots, n),
$$

be two matrices of analytic functions, each analytic in the neighborhood of a point $x=x_{0}$ but not necessarily analytic at the point $x_{0}$. If the matrix $M(x)$ defined by the matrix equation

$$
A(x)=M(x) B(x)
$$

is composed of elements $m_{i j}(x)$ each analytic at $x=x_{0}$ and if the determinant $|M(x)|$ of the matrix $M(x)$ is not zero at $x_{0}$, then $A(x)$ is equivalent to $B(x)$ at $x=x_{0}$ (from the left). $\ddagger$

From this definition it follows immediately that if $A(x)$ is equivalent to $B(x)$ at $x=x_{0}$, then $B(x)$ is also equivalent to $A(x)$; if, further, $B(x)$ is equivalent to $C(x)$, then $A(x)$ is equivalent to $C(x)$.

Two matrices equivalent to each other at a point have essentially the same type of singularity at the point. It is this fact which gives importance to the notion.

Evidently any two matrices of functions analytic at $x_{0}$ are equivalent if the determinants of the two matrices do not vanish at the point. For this reason a point for which the elements of a matrix are analytic, and the determinant

* Presented to the Society, December 28, 1915.

† See, for example, Proceedings of the American Academy of Arts a n d S c i e n ce s, vol. 49 (1913), pp. 521-568; in particular p. 540.

$\ddagger$ For the elements of the theory of matrices assumed in the present paper see Schlesinger, Vorlesungen über linearen Differentialgleichungen, pp. 18-19. 
of the matrix is not zero, will be called an ordinary point of the matrix. If the elements of the matrix are analytic, while the determinant vanishes, the matrix will be said to have a singular point of analytic type at $x=x_{0}$; if the determinant vanishes to the $k$ th order, this singular point will be said to be of multiplicity $k$.

If the elements have poles of the $k$ th order at most at $x=x_{0}$, the matrix will be said to have a pole of the kth order; at such a point the determinant of the matrix will be analytic or have a pole of order not greater than $n k$.

Finally, if the elements are analytic in the neighborhood of a given point while some elements have an essential singularity at the point, and if the determinant of the matrix does not vanish in the neighborhood of the point, the matrix will be said to have an isolated essentially singular point there; if, however, the determinant does vanish in every neighborhood of the point, or if the point under consideration is a cluster point of singular points of the matrix not of analytic type, the matrix will be said to have a non-isolated essentially singular point there.

It is to be recalled that in the theory of the factorization of an analytic function a cluster point of zeros does not play the rôle of an isolated essentially singular point.

\section{Factorization of polynomial matrices}

We will commence with the consideration of matrices whose elements are polynomials. By the order of such a matrix we shall mean the degree of its polynomial determinant in $x$.

From the standpoint of factorization the simplest type is that of order zero (constant determinant). The inverse of such a matrix and the product of two such are also of order zero. These matrices are not singular in the finite plane.*

A matrix of polynomials will have a single singular point of analytic type in the finite plane if its determinant is of the form $c\left(x-x_{0}\right)^{k}$. This point will lie at $x=x_{0}$ and be of multiplicity $k$.

An elementary matrix will be a matrix of the form

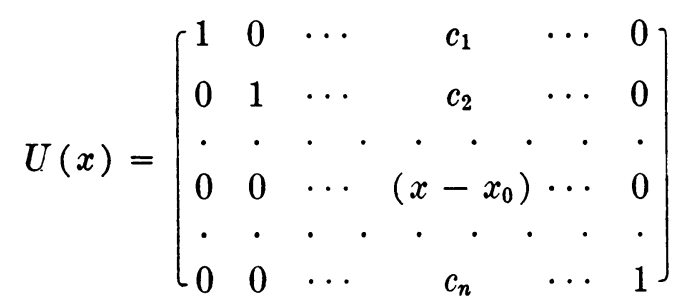

\footnotetext{
* Questions of factorization for such matrices have been considered by Hensel and Landsberg, Theorie der algebraischen Funktionen einer Variabeln, etc., p. 163.
} 
and differs from the unit matrix only in some $k$ th column where the successive elements are the following:

$$
c_{1}, c_{2}, \cdots, c_{k-1}, x-x_{0}, c_{k+1}, \cdots, c_{n} .
$$

Its only finite singular point will be one of analytic type and multiplicity 1 at $x=x_{0}$. The $k$ th column of the matrix $U(x)$ will be termed its characteristic column.

LEMMA. The most general matrix $F(x)$ with a singular point of analytic type at $x=x_{0}$ of multiplicity $k$ may be decomposed into the product of a matrix $Q(x)$, for which $x_{0}$ is an ordinary point if $k=1$ or a singular point of analytic type and of multiplicity $k-1$ if $k>1$, and a second elementary matrix $U(x)$ with singular point at $x_{0}, i . e$.,

$$
F(x)=Q(x) U(x) \text {. }
$$

Proof.-The determinant of $F\left(x_{0}\right)$ is zero by hypothesis and thus we may determine a matrix of constants $D$ of determinant not zero so that the product matrix $F\left(x_{0}\right) D$ has the elements of some $l$ th column all zero. To effect this it is necessary merely to determine the elements $d_{1 l}, d_{2 l}, \cdots, d_{n l}$ (not all zero) of that column of $D$ so that the $n$ equations

$$
f_{i 1}\left(x_{0}\right) d_{1 l}+f_{i 2}\left(x_{0}\right) d_{2 l}+\cdots+f_{i n}\left(x_{0}\right) d_{n l}=0 \quad(i=1,2, \cdots, n)
$$

hold, and then to choose the remaining elements of $D$ so that its deteminant does not vanish. But this set of $n$ linear homogeneous equations in $d_{1 l}$, $d_{2 l}, \cdots, d_{n l}$ can always be satisfied since the determinant of the coefficients is $\left|F\left(x_{0}\right)\right|=0$.

Furthermore let us assume for the moment that the first of these quantities $d_{1 l}$ is not zero and let us take $l=1$. In this case the elements of $D$ occurring in the $n-1$ columns after the first may be taken to be 1 along the principal diagonal and zero elsewhere. Also the elements of the first column may be taken to be $1, d_{2}, d_{3}, \cdots, d_{n}$ so that

$$
D=\left[\begin{array}{llll}
1 & 0 & \cdots & 0 \\
d_{2} & 1 & \cdots & 0 \\
\cdot & \cdot & \cdot & \cdot \\
d_{n} & 0 & \cdots & \cdot
\end{array}\right] .
$$

When $D$ has been taken in this way, the matrix $F(x) D$ has the property that the elements of the first column vanish at $x=x_{0}$. In consequence, if we write

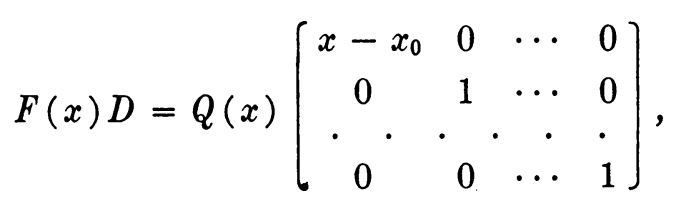


so that $Q(x)$ is derived from $F(x) D$ by dividing the elements of the first column of $F(x) D$ by $x-x_{0}$, the matrix $Q(x)$ is made up of elements analytic at $x_{0}$.

Moreover, if $k=1$, the determinant of $Q(x)$ is not zero at $x=x_{0}$; this follows from the determinant relation $|F(x)|=|Q(x)|\left(x-x_{0}\right)$. Likewise, if $k>1$, the same relation shows that $Q(x)$ has a singular point of analytic type of multiplicity $k-1$.

If we multiply the above matrix equation through by $D^{-1}$ on the right, we obtain

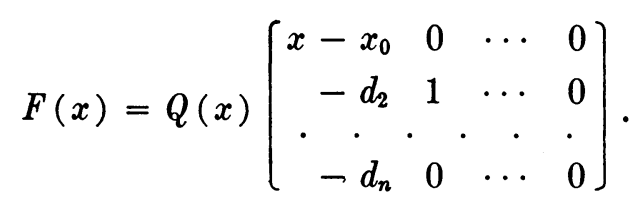

The second factor is evidently an elementary matrix $U(x)$ while the first factor $Q(x)$ has the properties demanded of it in the lemma. The first column of $U(x)$ is the characteristic column.

In the case when $d_{1 l}$ is zero some one of the quantities $d_{2 l}, \cdots, d_{n l}$ will be different from zero. If $d_{2 l}$ is not zero, for example, we may take $l=2$ and factor $F(x)$ into the product of a matrix $Q(x)$ and an elementary matrix $U(x)$ whose second column is its characteristic column. In general if the $\lambda$ th of the quantities $d_{1 l}, \cdots, d_{n l}$ is not zero we may take $l=\lambda$ and proceed as before.

This completes our proof of the lemma.

It is evident, in the simple case when $F(x)$ has a singular point of multiplicity one at $x=x_{0}$, that there may be $n$ distinct ways of separating out an elementary factor, namely by allowing $l$ to range from 1 to $n$. Hence the theory of factorization for $n>1$ diverges greatly from that for the case $n=1$ inasmuch as the mode of factorization is not unique.

The matrix $Q(x)$ of the lemma has the same singular points in the finite plane for $x \neq x_{0}$ as $P(x)$. Furthermore such a singularity is of the same kind for both matrices, i. e., is of analytic type with the same multiplicity, or a pole of order $k$, or an isolated essential singularity, or a non-isolated essential singularity for both. This is an obvious consequence of (2) and the inverse relation $Q(x)=F(x) U^{-1}(x)$.

We are now in a position to establish at once the following theorem:

Theorem I. An arbitrary matrix of polynomials $P(x)$ of order $m$ whose determinant is factorable into the product

$$
c\left(x-x_{1}\right)\left(x-x_{2}\right) \cdots\left(x-x_{m}\right) \quad(c \neq 0)
$$

may be expressed as a product 


$$
P_{0}(x) U_{1}(x) U_{2}(x) \cdots U_{m}(x)
$$

where $P_{0}(x)$ is a polynomial matrix of order zero and where $U_{1}(x), U_{2}(x)$, $\cdots, U_{m}(x)$ are elementary matrices with singular points at $x_{1}, x_{2}, \cdots, x_{m}$ respectively.

It is obvious that a successive application of the above lemma enables us to write $P(x)$ in the stated form.

Suppose now that a polynomial matrix $P(x)$ be expressed in any way as a product $V_{1}(x) V_{2}(x) \cdots V_{m}(x)$ of $m$ polynomial matrices $V_{1}(x), V_{2}(x)$, $\cdots, V_{m}(x)$, each having its only finite singularity at distinct points $x_{1}, x_{2}$, $\cdots, x_{m}$ respectively. A particular product of this sort can be obtained at once from the product for $P(x)$ of Theorem I.

The most general factored form $\bar{V}_{1}(x) \bar{V}_{2}(x) \cdots \bar{V}_{m}(x)$ of $P(x)$ with the same properties is defined by the equations

$$
\begin{aligned}
& \bar{V}_{1}(x)=V_{1}(x) R_{1}(x), \\
& \bar{V}_{2}(x)=R_{1}^{-1}(x) V_{2}(x) R_{2}(x) \text {, } \\
& \bar{V}_{m}(x)=R_{m-1}^{-1}(x) V_{m}(x),
\end{aligned}
$$

where $R_{1}(x), \cdots, R_{m-1}(x)$ are arbitrary polynomial matrices of order zero.

It is clear that the indicated general factored form is a possible form.

Further, any factorization $\bar{V}_{1}(x) \bar{V}_{2}(x) \cdots \bar{V}_{m}(x)$ leads at once to the matrix identity

$$
V_{2}(x) \cdots V_{m}(x) \bar{V}_{m}^{-1}(x) \cdots \bar{V}_{2}^{-1}(x)=V_{1}^{-1}(x) \bar{V}_{1}(x) .
$$

The matrix $R_{1}(x)$, as given by the right-hand member, has its only possible finite singularity at $x_{1}$, while, as given by the left-hand member, it has its only possible singularities at $x_{2}, \cdots, x_{m}$. Hence $R_{1}(x)$ is not singular in the finite plane and must be composed of polynomial elements. This gives us the first equation.

By use of the relation just obtained we find

$$
V_{2}(x) \cdots V_{m}(x)=R_{1}(x) \bar{V}_{2}(x) \cdots \bar{V}_{m}(x),
$$

and from this we get further

$$
V_{3}(x) \cdots V_{m}(x) \bar{V}_{m}^{-1}(x) \cdots V_{3}^{-1}(x)=V_{2}^{-1}(x) R_{1}(x) \bar{V}_{2}(x) .
$$

The matrix given by either side of this equation must, for a similar reason, be a polynomial matrix $R_{2}(x)$ of order zero. This conclusion leads us at once to the second of the given equations.

Continuing in this way we derive all of the desired equations in succession.

It is interesting to consider a particular resolution in elementary factors 
possible in general when the elements of $P(x)$ are polynomials of the $\mu$ th degree and when $|P(x)|$ is a polynomial of degree $n \mu=m$ with distinct roots $x_{1}, x_{2}, \cdots, x_{m}$.

If we remove an elementary factor corresponding to $x=x_{m}$ for which the first column is the characteristic column, we thereby only alter the first column of $P(x)$. In fact we obtain the quotient $Q(x)$ of the lemma from $P(x)$ by multiplying on the right by $U^{-1}(x)$, which is equivalent to adding a constant multiple of the second, third, $\cdots$, last column to the first column, and dividing that column by $x-x_{m}$. Thus the degree of the first column is lowered by unity, while the other columns are unaltered.

In the same way we may lower the degree of the second column by unity, by the removal of an elementary factor corresponding to $x=x_{m-1}$ for which the second column is the characteristic column.

By this process, after $n$ steps, we lower the degree of all $n$ columns of $P(x)$ by unity by removing $n$ elementary factors.

Repeating the process we can reduce the quotient to a constant matrix in $n \mu$ steps. Every step can be carried out in the general case, i. e., if certain particular algebraic relations between the coefficients of $P(x)$ do not hold.

Thus the matrix $P_{0}(x)$ of Theorem I of order zero can be taken to be a matrix of constants in this case.

This result shows that one can not expect to obtain a simpler normal-form for a factor with one singular point than the elementary matrix. For we have used the $n \mu$ roots $x_{1}, \cdots, x_{m}$, the $n \mu$ elementary factors each involving $n-1$ arbitrary constants, and a constant matrix with $n^{2}$ constants. Thus there are involved $n^{2}(\mu+1)$ explicit constants in the factorization of $P(x)$, precisely as many as there are in the polynomial matrix $P(x)$. No further simplification can be expected since no superfluous constants have been employed.

\section{Equivalence PROBlem FOR POLYNomial Matrices}

In the first paragraph we solved the simple problem of factorization of polynomial matrices. The converse problem of constructing a polynomial matrix with given factors is solved merely by writing down the product of the factors.

But it is possible to modify the point of view slightly. Every polynomial matrix with singularities at the distinct points $x_{1}, \cdots, x_{m}$ is by the lemma of $\S 1$ equivalent to a polynomial matrix $V_{i}(x)$ at the point $x_{i}$ whose only finite singularity lies at $x_{i}$ for $i=1,2, \cdots, m$.

We are thus led to formulate a converse problem which may be stated as follows:

EQUivalenCe PROBlem. Given polynomial matrices $V_{1}(x), \cdots, V_{m}(x)$, each having its only finite singular point at the distinct points $x_{1}, \cdots, x_{m} r e-$ 
spectively; to construct a polynomial matrix $P(x)$ equivalent to these matrices at $x_{1}, \cdots, x_{m}$ respectively but with no finite singular points other than $x_{1}, \cdots, x_{m}$.

Before proceeding to its solution we may note that the formulation of the problem is symmetrical with respect to all the singular points involved.

An explicit solution may be obtained by the aid of a simple notation. Let $[T(x)]_{\xi}$ denote a polynomial matrix, with its only finite singularity at $\xi$, to which the polynomial matrix $T(x)$ is equivalent at the singular point $x=\xi$ of $T(x)$. The lemma of factorization of $\S 1$ shows that, since $T(x)$ is composed of elements analytic at $x=\xi$, a matrix $[T(x)]_{\xi}$ given as a product of elementary matrices, will always exist. If now we write for brevity

a solution is

$$
\begin{aligned}
& P_{1}(x)=\left[V_{1}(x)\right]_{x_{1}}=V_{1}(x), \\
& P_{2}(x)=\left[V_{2}(x) P_{1}^{-1}(x)\right]_{x_{2}} \text {, } \\
& P_{m}(x)=\left[V_{m}(x) P_{1}^{-1}(x) \cdots P_{m-1}^{-1}(x)\right]_{x_{m}},
\end{aligned}
$$

$$
P(x)=P_{m}(x) P_{m-1}(x) \cdots P_{1}(x) .
$$

To prove that $P(x)$, as defined in this manner, has the desired properties we begin by observing that $P(x)$ is certainly equivalent to $V_{1}(x)$ at $x=x_{1}$ since the product $P_{m}(x) \cdots P_{2}(x)$ is not singular at $x=x_{1}$.

Next let us proceed to the point $x_{2}$. By definition of $P_{2}(x)$ we may write

$$
P_{2}(x)=A(x) V_{2}(x) P_{1}^{-1}(x)
$$

where $A(x)$ is not singular at $x=x_{2}$. Consequently, on substituting in this value for $P_{2}(x)$, we see that

$$
P(x)=P_{m}(x) \cdots P_{3}(x) A(x) V_{2}(x) .
$$

But $P_{m}(x), \cdots, P_{3}(x)$ and $A(x)$ are not singular at $x=x_{2}$ so that $P(x)$ is evidently equivalent to $V_{2}(x)$ at $x=x_{2}$.

In precisely the same way we proceed with the proof that $P(x)$ is equivalent to $V_{3}(x), \cdots, V_{m}(x)$ at $x_{3}, \cdots, x_{m}$ respectively, and thus forms a solution.

The most general solution $\bar{P}(x)$ of the equivalence problem is $P_{0}(x) P(x)$ where $P_{0}(x)$ is any polynomial matrix of order zero.

In the first place it is clear that $\bar{P}(x)$ as so defined is a solution of the equivalence problem. Moreover, consider any solution $\widetilde{P}(x)$. At any singular point as $x_{1}$ we have

$$
\begin{aligned}
& P(x)=A(x) V_{1}(x), \\
& \tilde{P}(x)=B(x) V_{1}(x),
\end{aligned}
$$

where $A(x)$ and $B(x)$ are not singular at $x=x_{1}$. Using these values of 
$P$ and $\tilde{P}$ we conclude that the matrix $\tilde{P}(x)[P(x)]^{-1}$ is equal to $B(x) A^{-1}(x)$ and thus is not singular at $x_{1}$. Similarly this matrix is not singular at $x_{2}$, $\cdots, x_{m}$ and also of course at any other point of the finite $x$-plane. Since $\tilde{P}(x)$ by hypothesis is a polynomial matrix the matrix $P_{0}(x)$ under consideration must be a polynomial matrix of order zero, which we were to prove.

\section{Factorization of matrices of entire functions. First method}

Let $E(x)$ be a matrix of entire functions. Its finite singularities are all of analytic type, and fall at the points $x_{1}, x_{2}, \cdots$, at which the determinant of $E(x)$ vanishes. We take these points to be arranged in some arbitrary order and assume that they are infinitely many in number. It is clear that we have $\lim x_{m}=\infty$.

Suppose that $V_{1}(x)$ is some polynomial matrix with unique finite singularity at $x_{1}$, to which $E(x)$ is equivalent at $x_{1}$; for instance $V_{1}(x)$ may be a product of elementary matrices with singularity at $x_{1}$, as follows from the lemma of $\S 1$. The matrix $E(x) V_{1}^{-1}(x)$ is a matrix of entire functions without a singularity at $x=x_{1}$ but with its other finite singularities at $x_{2}, x_{3}, \ldots$. At $x_{2}$ this modified matrix is equivalent to a polynomial matrix $V_{2}(x)$ with unique finite singularity at $x_{2}$. Forming now the matrix of entire functions $E(x) V_{1}^{-1}(x) V_{2}^{-1}(x)$, which is not singular at $x_{1}$ and $x_{2}$, we are led to $V_{3}(x)$, $V_{4}(x), \cdots$ in similar fashion.

It is therefore possible to find such polynomial matrices $V_{1}(x), V_{2}(x)$, ... that $E(x)$ is equivalent to $V_{1}(x)$ at $x_{1}$, to $V_{2}(x) V_{1}(x)$ at $x_{2}$, and so on. The following theorem shows that matrices $E(x)$ of entire functions having this property exist for any choice of $V_{1}(x), V_{2}(x), \cdots$ with a single finite singularity of analytic type at $x_{1}, x_{2}, \cdots$ respectively.

Theorem II. If $V_{1}(x), V_{2}(x), \cdots$ are polynomial matrices each with a single finite singularity of analytic type at the distinct points $x_{1}, x_{2}, \cdots$ and if $\lim x_{m}=\infty$, there exists a matrix of entire functions equivalent to the product $V_{m}(x) \cdots V_{1}(x)$ at $x_{m}(m=1,2, \cdots)$ and of the form

$$
E(x)=\lim _{m=\infty} Q_{m}(x) V_{m}(x) \cdots V_{1}(x),
$$

where $Q_{m}(x)(m=1,2, \cdots)$ is a matrix of entire functions without singularities in the finite plane.

Proof. Let us first attempt to choose $Q_{1}(x), Q_{2}(x), \cdots$ so that the sequence of matrices

$$
R_{m}(x)=Q_{m}(x) V_{m}(x) \cdots V_{1}(x)
$$

converges to a limit matrix as $m$ approaches infinity. Now we have clearly

$$
R_{m+1}(x)=\left[I+S_{m}(x)\right]\left[I+S_{m-1}(x)\right] \cdots\left[I+S_{1}(x)\right] Q_{1}(x),
$$

where $I+S_{m}(x)=Q_{m+1}(x) V_{m+1}(x) Q_{m}^{-1}(x)$. 
It will therefore suffice to establish that the matrices $S_{m}(x)$ can be made to have elements of as small absolute value as desired within a circle of radius less than $x_{m}$ with center at the origin. This will be established by means of the following lemma:

Lемма. If $F(x)$ be a matrix without singularity in some closed continuum $C$ in the finite plane, it is possible to find a matrix $F_{1}(x)$ without singularity in the finite plane and such that the absolute values of the elements of the difference matrix $F(x)-F_{1}(x)$ are arbitrarily small throughout $C$.

A very simple proof of the lemma may be given as follows. The elements of the matrix

$$
\frac{d F(x)}{d x} F^{-1}(x)=A(x)
$$

are not singular in $C$. The elements $a_{i j}(x)$ of $A(x)$ may then be represented by series of polynomials which converge uniformly throughout $C$, by a wellknown theorem due to Runge.* Hence we may break off the terms of these series at such a place that the elements of the resulting matrix $A_{1}(x)$ differ from those of $A(x)$ by a uniformly small quantity throughout $C$.

Consider now the matrix differential equation

$$
\frac{d F_{1}(x)}{d x}=A_{1}(x) F_{1}(x)
$$

By comparison with the equation for $F$,

$$
\frac{d F(x)}{d x}=A(x) F(x),
$$

we infer that a solution $F_{1}(x)$ of the preceding equation may be found whose elements are analytic and differ from those of $F(x)$ by as little as we please throughout the given continuum $C$.

But the differential equation for $F_{1}$ is without finite singular points so that $\left|F_{1}(x)\right|$ nowhere vanishes. $\dagger$ Hence the matrix $F_{1}(x)$ has the desired properties.

Let us now return to the proof of the theorem. Suppose that the matrices $Q_{1}, \cdots, Q_{m}$ have been so selected that the matrices $S_{1}, \cdots, S_{m-1}$ determined by this choice have their elements in absolute value less than assigned positive quantities $a_{1}, \cdots, a_{m-1}$ respectively within given circles of radii less than $\left|x_{2}\right|, \cdots,\left|x_{m}\right|$. We shall now show that $Q_{m+1}$ can then be so selected that the corresponding matrix $S_{m}$ has its elements less in absolute value than a prescribed positive quantity $a_{m}$ within any given circle of radius less than $\left|x_{m+1}\right|$.

*A ct a M a the matica, vol. 6 (1884), p. 229.

† Schlesinger, loc. cit., p. 23. 
To this end we observe that the matrix

$$
Q_{m}(x) V_{m_{+}}^{-1}(x)
$$

will not be singular except at $x=x_{m+1}$. Thus, by the lemma above, a matrix $Q_{m+1}(x)$ without finite singularities may be chosen so that the difference matrix

$$
Q_{m+1}(x)-Q_{m}(x) V_{m+1}^{-1}(x)
$$

has elements of as small absolute value as desired for $|x| \leqq d_{m}<\left|x_{m+1}\right|$. Hence the same is true of

$$
S_{m}(x)=Q_{m+1}(x) V_{m+1}(x) Q_{m}^{-1}(x)-I,
$$

as we see by multiplying the preceding expression on the right by $V_{m+1}(x) Q_{m}^{-1}(x)$.

Thus we have a method of assigning $Q_{m+1}(x)$ as soon as $Q_{m}(x)$ has been chosen, in such a way that the elements of $S_{m}$ are less than a positive constant $a_{m}$ for $|x|<d_{m}<\left|x_{m+1}\right|$, where $a_{1}+a_{2}+\cdots$ is a convergent series. Moreover, since $\lim x_{m}=\infty$ we may take $\lim d_{m}=\infty$ also.

But the elements of the infinite sequence of matrix products

$$
\begin{array}{r}
R_{m+1}(x) Q_{1}^{-1}(x)=\left[I+S_{m}(x)\right]\left[I+S_{m-1}(x)\right] \cdots\left[I+S_{1}(x)\right] \\
(m=1,2, \cdots)
\end{array}
$$

will certainly converge absolutely and uniformly to the elements of a limit matrix if the elements of $S_{m}(x)$ are less in absolute value than the $m$ th term of a convergent series of positive terms. ${ }^{*}$ Furthermore the determinant of this limit matrix is the limit of the product of the determinants of the factors and the sum of the logarithms of these factors will form a convergent series. Hence we infer that $\lim R_{m}(x)$ is a matrix of entire functions $E(x)$ of the form (5) and of determinant not zero save at $x_{1}, x_{2}, \cdots$ at which points $E(x)$ is equivalent to $V_{1}(x), V_{2}(x) V_{1}(x), \cdots$ respectively, so that $E(x)$ has the desired properties.

The most general matrix $E(x)$ of entire functions with its singularities at $x_{1}, x_{2}, \cdots$ and equivalent to $V_{1}(x), V_{2}(x) V_{1}(x), \cdots$ at these points respectively has the form $Q(x) E(x)$ where $Q(x)$ is a matrix of entire functions with no finite singular point. This fact is immediately evident.

In the case $n=1$ the matrix $Q_{m}(x)$, as determined by the method of the lemma, is merely an exponential with an exponent which is polynomial in $x$. Thus we have a thoroughgoing generalization of the factorization theorem in the case $n=1$, but one which appears much less explicit. Furthermore, the determination of each convergence factor $Q_{m+1}(x)$ is made to depend on the previous factor $Q_{m}(x)$.

\footnotetext{
* The proof for $n>1$ is not essentially more difficult than in the familiar case $n=1$.
} 
4. Factorization of matrices of entire functions. Second method

A more explicit form of factorization can be given by the aid of an extended elementary matrix

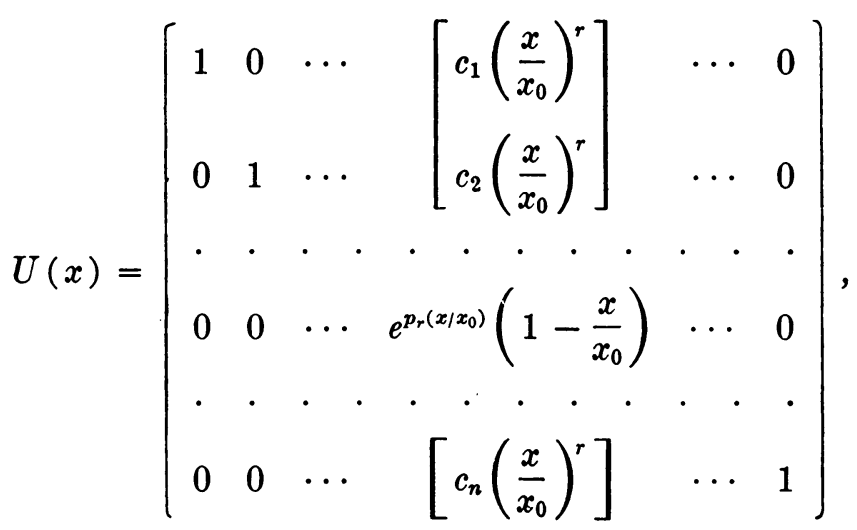

where

$$
p_{r}(x)=x+\frac{x^{2}}{2}+\frac{x^{3}}{3} \cdots+\frac{x^{r}}{r} .
$$

This matrix differs from the unit matrix only in some $l$ th column and reduces essentially to an elementary matrix for $r=0$ (compare with (1)).

It is apparent that for a given value of $r$ the lemma of $\S 1$ may be extended to this type of matrix; i. e., a matrix $F(x)$ with singularity of analytic type at $x=x_{0}$ of multiplicity $k$ may be written as $Q(x) U(x)$ where $Q$ has its elements analytic at $x_{0}$, and has a singularity of analytic type of order $k-1$ at $x=x_{0}$ if $k>1$, or is not singular at $x_{0}$ if $k=1$.

In fact, if we multiply $F(x)$ on the right by a matrix

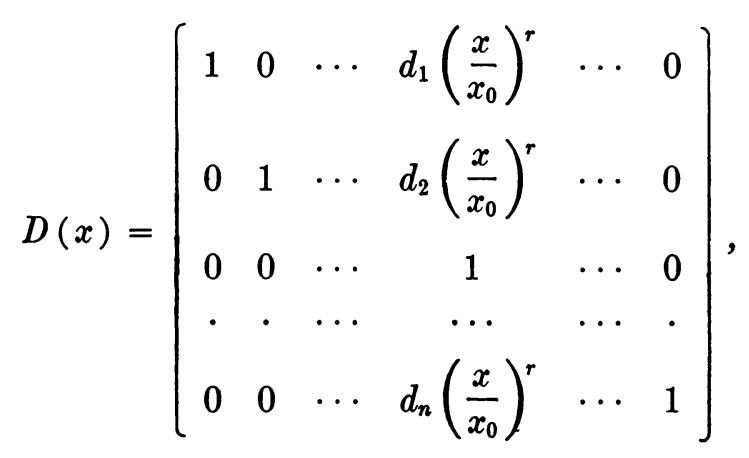

which takes the same form as $D$ of $\S 1$ for $x=x_{0}$ and in which the $l$ th column contains the $d$ 's, we may reduce the elements of the lth column of $F(x)$ to zero at $x=x_{0}$ (for some $l$ ). Consequently if we write 


$$
F(x) D(x)=Q(x)\left[\begin{array}{cccccc}
1 & 0 & \ldots & 0 & \cdots & 0 \\
0 & 1 & \ldots & 0 & \cdots & 0 \\
. & . & \cdots & \cdots & \cdots & . \\
0 & 0 & \cdots & e^{p_{r}\left(x / x_{0}\right)}\left(1-\frac{x}{x_{0}}\right) & \cdots & 0 \\
. & . & \ldots & \ldots & \cdots & . \\
0 & 0 & \ldots & 0 & \cdots & 1
\end{array}\right]
$$

the elements of $Q(x)$ have the desired properties, since $Q(x)$ is derived from $F(x) D(x)$ merely by dividing the $l$ th column by

$$
e^{p_{r}\left(x / x_{0}\right)}\left(1-\frac{x}{x_{0}}\right)
$$

Multiplying through the above equation by the inverse of $D(x)$ we have $F(x)$ expressed as the product of $Q(x)$ and an extended elementary matrix $U(x)$ like $(7)$ with $c_{1}=-d_{1}, c_{2}=-d_{2}, \cdots, c_{n}=-d_{n}$.

It is to be especially observed that the choice of the quantities $d_{i}$ and accordingly $c_{i}$ is independent of the choice of $r$ which does not enter explicitly into $D(x)$ for $x=x_{0}$.

We are now prepared to demonstrate immediately the following second factorization theorem for matrices of entire functions:

Theorem III. Any matrix of entire functions $E(x)$ with its singularities at the distinct points $x_{1}, x_{2}, \cdots$ may be written in the form

$$
E_{0}(x) \lim _{m=\infty} U_{m}(x) U_{m-1}(x) \cdots U_{1}(x)
$$

where $E_{0}(x)$ is a matrix of entire functions of determinant nowhere zero and where $U_{1}(x), U_{2}(x), \cdots$ are extended elementary matrices of the form (7) such that the finite singular point of $U_{1}(x), U_{2}(x), \cdots, U_{k}(x)$ lies at $x_{1}$, that of $U_{k+1}(x), \cdots U_{l}(x)$ lies at $x_{2}$, and so on.

Proof. The possibility of selecting extended elementary matrices $U_{1}(x)$, $\cdots, U_{k}(x)$ with singular point at $x_{1}$, such that the product $U_{k}(x) \cdots U_{1}(x)$ is equivalent to $E(x)$ at $x=x_{1}$, is immediately obvious. In fact we may determine the product by successive application of the extended form of the lemma of $\S 1$, so that it is equivalent to $E(x)$ at $x_{1}$. Consider now the product

$$
E(x)\left[U_{k}(x) \cdots U_{1}(x)\right]^{-1},
$$

which is a matrix of entire functions with its finite singular points at $x_{2}$, $x_{3}, \cdots$. Treating this matrix at $x_{2}$ as we did $E(x)$ at $x_{1}$, we can find a second product of the form $U_{k+1}(x) \cdots U_{l}(x)$ to which the product matrix under 
consideration is equivalent at $x_{2}$. Proceeding in this way we obtain a sequence of matrices $U_{1}(x), U_{2}(x), \cdots$.

We can, in addition, secure the uniform convergence of the elements of the product matrix

$$
U_{m}(x) U_{m-1}(x) \cdots U_{1}(x),
$$

for $\lim m=\infty$, within any arbitrary circle $|x|=\rho$ in the complex plane.

For we have noted that, at each step in the determination of the successive factors, the constants $c_{1}, c_{2}, \cdots, c_{n}$ are chosen independently of the integer $r$. Thus, at each step after these constants have been chosen, we can take $r$ so large that the elements of the $l$ th column of that factor,

$$
c_{1}\left(\frac{x}{x_{k}}\right)^{r}, c_{2}\left(\frac{x}{x_{k}}\right)^{r}, \cdots, e^{p_{r}\left(x / x_{k}\right)}\left(1-\frac{x}{x_{k}}\right), \cdots, c_{n}\left(\frac{x}{x_{k}}\right)^{r},
$$

differ from the corresponding elements of the set

$$
0,0, \cdots, 1, \cdots 0
$$

within any assigned circle $|x|=\rho<\left|x_{k}\right|$ by an arbitrarily small amount. Hence the elements of the corresponding factor $U_{m}(x)$ can be made to differ from the elements of the unit matrix by arbitrarily small quantities throughout this circle.

It is therefore apparent that the factors $U_{1}(x), U_{2}(x), \cdots$ can be so taken that the limit of their product exists uniformly within every fixed circle with center at the origin in the complex plane. The elements of this limit matrix $E_{1}(x)$ are of course entire functions, and it has the desired equivalence properties at $x_{1}, x_{2}, \cdots$. The matrix $E_{0}(x)$ defined by the equation

$$
E(x)=E_{0}(x) E_{1}(x)
$$

is a matrix not singular in the finite plane.

\section{Equivalence problem for matrices of entire functions}

The results of $\$ 3$ permit us to solve the following problem:

Equivalence PRoblem. Given polynomial matrices $V_{1}(x), V_{2}(x), \cdots$ each with its only finite singular point at the distinct points $x_{1}, x_{2}, \cdots$ respectively; to construct a matrix $E(x)$ of entire functions equivalent to $V_{1}(x)$, $V_{2}(x), \cdots$ at $x_{1}, x_{2}, \cdots$ respectively but with no other finite singular points.

In fact, let us use the bracket notation $[T(x)]_{\xi}$ as before to denote some polynomial matrix equivalent to $T(x)$ at $x=\xi$ but otherwise not singular in the finite plane. Let us write 


$$
\begin{aligned}
& \bar{V}_{1}(x)=\left[V_{1}(x)\right]_{x_{1}}=V_{1}(x), \\
& \bar{V}_{2}(x)=\left[V_{2}(x) \bar{V}_{1}^{-1}(x)\right]_{x_{2}}, \\
& \bar{V}_{3}(x)=\left[V_{3}(x) \bar{V}_{1}^{-1}(x) \bar{V}_{2}^{-1}(x)\right]_{x_{3}},
\end{aligned}
$$

Consider now the matrix of entire functions

$$
E(x)=\lim _{m=\infty} Q_{m}(x) \bar{V}_{m}(x) \cdots \bar{V}_{1}(x),
$$

where the matrices $Q_{m}(x)$ are chosen as in Theorem II so that the limit is approached uniformly. It is readily seen that the product $\bar{V}_{m}(x) \cdots \bar{V}_{1}(x)$ is equivalent to $V_{i}(x)$ at $x=x_{i}$ for $i=1,2, \cdots, m$. This is obvious for $i=1$. Furthermore at $x=x_{2}$ we may write

$$
\bar{V}_{2}(x)=A(x) V_{2}(x) \bar{V}_{1}^{-1}(x)
$$

where $A(x)$ is not singular at $x=x_{2}$. It follows at once that $\bar{V}_{2}(x) \bar{V}_{1}(x)$ and hence $\bar{V}_{m}(x) \cdots \bar{V}_{1}(x)$ is equivalent to $V_{2}(x)$ at $x=x_{2}$. Continuing in this way we may establish the truth of the statement made for $i=1,2$, $\cdots, m$.

The definition of $E(x)$ now shows that it has the stated properties. Thus the equivalence problem has been solved.

The most general solution of the equivalence problem is $E_{0}(x) E(x)$ where $E_{0}(x)$ is an arbitrary matrix of entire functions of determinant not zero in the finite plane.

\section{Matrices With a Finite NUMBER OF ESSENTIAL SINGUlarities}

Thus far we have restricted attention to matrices with singular points of analytic type. We propose now to consider the question of the factorization of matrices with isolated essential singularities. We will restrict attention at first to the case in which there are only a finite number of isolated essentially singular points.

I shall assume the truth of the following lemma:

LEMma. Let $L(x)$ be a matrix of functions for which $x=\infty$ is an isolated essentially singular point. Then it is possible to write $L(x)$ in the product form $A(x) E(x)\left(x^{k_{j}} \delta_{i j}\right)$ where $A(x)$ is a matrix of functions not singular at $x=\infty$, where $E(x)$ is a matrix of entire functions not singular in the finite plane, and where $k_{1}, k_{2}, \cdots, k_{n}$ are integers.

For the proof of this lemma the reader is referred to the Mathe matische Annalen, vol. 74 (1913), pp. 122-133. With its aid we may at once prove the theorem which follows:

Theorem IV. Any matrix $F(x)$ with a finite number of isolated singularities 
(essential or non-essential) at the distinct points $x_{1}, x_{2}, \cdots, x_{m}, x_{m+1}=\infty$, and no others, can be written as a product of factors $E(x) W_{m}(x) W_{m-1}(x) \cdots$ $W_{1}(x)$ where these factors $W$ are

$$
W_{l}(x)=E_{l}\left(\frac{1}{x-x_{l}}\right)\left[\left(x-x_{l}\right)^{k_{j}^{(l)}} \delta_{i j}\right] .
$$

Here $E(y), E_{m}(y), \cdots, E_{1}(y)$ are matrices of entire functions not singular in the finite plane and $k_{j}^{(l)}(l=1,2, \cdots, m ; j=1,2, \cdots, n)$ are integers.

Proof. It is clear that $F\left[\left(x_{1}+1 / \xi\right)\right]$ has an isolated essential singularity at $\xi=\infty$ and so, by the above lemma, may be written as

$$
A_{1}(\xi) E_{1}(\xi)\left(\xi^{k_{j}^{(1)}} \delta_{i j}\right),
$$

where $E_{1}(\xi)$ is a matrix of entire functions which is not singular in the finite plane. Transferring back to the variable $x$, we obtain the decomposition

$$
F(x)=F_{1}(x) E_{1}\left(\frac{1}{x-x_{1}}\right)\left[\left(x-x_{1}\right)^{-k_{j}^{(1)}} \delta_{i j}\right],
$$

where $F_{1}(x)$ is not singular at $x=x_{1}$. It follows that $F_{1}(x)$ has singularities only at $x_{2}, \cdots, x_{m}$, and $x_{m+1}=\infty$.

By the same process we find

$$
F_{1}(x)=F_{2}(x) E_{2}\left(\frac{1}{x-x_{2}}\right)\left[\left(x-x_{2}\right)^{-k_{j}^{(2)}} \delta_{i j}\right],
$$

where $F_{2}(x)$ is not singular save at $x_{3}, \cdots, x_{m}$, and $x_{m+1}=\infty$.

Proceeding successively in this manner we obtain a set of similar equations by introducing matrices $F_{3}, \cdots, F_{m}$. The last equation is

$$
F_{m-1}(x)=F_{m}(x) E_{m}\left(\frac{1}{x-x_{m}}\right)\left[\left(x-x_{m}\right)^{-k_{j}^{(m)}} \delta_{i j}\right],
$$

where $F_{m}(x)$ is not singular save at infinity, and hence is a matrix $E(x)$ of entire functions of the desired type.

From this succession of equations we get the stated decomposition at once. It is not difficult to infer that the above decomposition is essentially unique.

\section{The CORRESPoNdING EQUIVALENCE PROBLEM}

We can now pass at once to the solution of the corresponding problem:

Equivalence PRoblem.* Given matrices $G_{1}(x), \cdots, G_{m}(x)$ each with one isolated singularity at the distinct points $x_{1}, \cdots, x_{m}$ respectively in the finite plane and with no other singular points; to construct a matrix $F(x)$ equivalent to

* Cf. Proceedings of the American Academy of Artsand Scie n c e s, vol. 49 (1913), pp. 521-568; in particular pp. 540-541. 
matrices $G_{1}(x), \cdots, G_{m}(x)$ at $x_{1}, \cdots, x_{m}$ respectively, and otherwise not singular save for a possible pole at infinity.

Proof. We follow the method used in $\$ \S 2,5$ in solving the similar problem for matrices of polynomials and entire functions.

Suppose that $T(x)$ has an isolated singularity at $x=\xi$. Let $[T(x)]_{\xi}$ be used to designate the matrix

$$
E\left(\frac{1}{x-\xi}\right)\left[(x-\xi)^{-k_{j}} \delta_{i j}\right]
$$

to which, in accordance with the lemma of the preceding section, $F(x)$ is equivalent at $x=\xi$. This matrix has no other singularity save for a possible pole at $x=\infty$. Here $E(y)$ is an entire function of $y$ and not singular in the finite plane.

If now we write

$$
\begin{aligned}
H_{1}(x) & =\left[G_{1}(x)\right]_{x_{1}}=G_{1}(x), \\
H_{2}(x) & =\left[G_{2}(x) H_{1}^{-1}(x)\right]_{x_{2}}, \\
\cdot & \cdot \cdot \cdot \cdot \cdot \cdot \cdot \cdot \cdot \cdot \cdot \\
H_{m}(x) & =\left[G_{m}(x) H_{1}^{-1}(x) \cdots H_{m-1}^{-1}(x)\right]_{x_{m}},
\end{aligned}
$$

we infer at once that the matrix $F(x)$ defined by

$$
F(x)=H_{m}(x) \cdots H_{1}(x)
$$

has the stated properties (see $\S 2$ ).

The most general solution of the equivalence problem is $Q(x) F(x)$, where $Q(x)$ is any polynomial matrix of constant determinant.

\section{The MatriX With General SINGUlarities}

The factorization theorems of $\$ \S 1,3$, admit of a further extension as follows:

Theorem V. Let $x_{1}, x_{2}, \cdots$ be an isolated set of points in the finite complex $p$ ane with a set of limit points [c], one of which lies at infinity in the plane. Further, let $\bar{E}_{1}(y), \bar{E}_{2}(y), \cdots$ be. arbitrary matrices of functions of the form $E(y)\left(y^{k_{j}} \delta_{i j}\right)$ where $E(y)$ is a matrix of entire functions not singular in the finite plane, and $k_{1}, \cdots, k_{n}$ are integers. Then there exists a matrix $G(x)$ of analytic functions given by a formula

$$
G(x)=\lim _{m=\infty} Q_{m}(x) \bar{E}_{m}\left(\frac{1}{x-x_{m}}\right) \cdots \bar{E}_{1}\left(\frac{1}{x-x_{1}}\right),
$$

where $Q_{m}(x)$ is not singular in the finite plane, such that $G(x)$ is equivalent to $\bar{E}_{m}\left[1 /\left(x-x_{1}\right)\right] \cdots \bar{E}_{1}\left[1 /\left(x-x_{2}\right)\right]$ at $x_{m}(m=1,2, \cdots)$ and is otherwise not singular save at the points of $[c]$. 
Proof. We shall follow closely the method used in proving the analogous Theorem II ( $\$ 3)$.

For convergence of a formula for $G(x)$ of the type indicated, it is sufficient to establish that $Q_{m+1}(x)$ can be so chosen that, in a region outside of an arbitrary small distance of $x_{m+1}$ and within a circle of arbitrarily large radius with center at this point in the complex plane, the elements of

$$
Q_{m+1}(x) \bar{E}_{m+1}\left(\frac{1}{x-x_{m}}\right) Q_{m}^{-1}(x)
$$

can be made to differ in absolute value from the corresponding elements of the unit matrix by less than an arbitrarily assigned positive quantity.

But this is readily done as follows. The matrix

$$
Q_{m}(x) \bar{E}_{m+1}^{-1}\left(\frac{1}{x-x_{m+1}}\right)
$$

is obviously not singular except at $x_{m+1}$ and at infinity. Hence by the lemma of $\S 3$ we can find a matrix $Q_{m+1}(x)$, not singular in the finite plane, whose elements differ from those of the above matrix by less than an arbitrarily assigned quantity for

$$
r \leqq\left|x-x_{m+1}\right| \leqq R,
$$

$r, R$ being arbitrary positive quantities.

Consequently we may make the elements of the matrix (9) differ from those of the unit matrix by less than an arbitrary positive quantity in this region for $r$ as small and $R$ as large as desired.

It is therefore clear that $Q_{1}(x), Q_{2}(x), \cdots$ may be taken in succession so that the limit in the theorem exists uniformly so long as $x$ lies within any circle of arbitrarily large radius with center at the origin and not within an arbitrarily small distance of any point of $[c]$.

The matrix $G(x)$ thus defined has clearly the desired property. On the basis of this theorem we are led at once (as in $\$ 5$ ) to a solution of the problem:

Equivalence PRoblem. Given an infinite number of points $x_{1}, x_{2}, \cdots$ in the finite plane with a set of limit points [c], one of which lies at infinity, and matrices $\bar{E}_{1}(y), \bar{E}_{2}(y), \cdots$ of the form $E(y)\left(y^{k_{j}} \delta_{i j}\right)$ where $E(y)$ is a matrix of entire functions, not singular in the finite plane, and $k_{1}, \cdots, k_{n}$ are integers; to construct a matrix $G(x)$ equivalent to $\bar{E}_{1}\left[1 /\left(x-x_{1}\right)\right], \bar{E}_{2}\left[1 /\left(x-x_{2}\right)\right], \cdots$ at $x_{1}, x_{2}, \cdots$ respectively, and otherwise not singular save at the points of $[c]$.

The most general solution of this equivalence problem is $M(x) G(x)$ where $M(x)$ is an arbitrary matrix of analytic functions whose singularities all fall at the points of $[c]$. 


\section{An application}

I shall give now a simple application of the preceding theorems. This application may be stated as follows: Let $e(x)$ be an entire function and let $e_{0}(x), e_{1}(x), e_{2}(x), e_{3}(x), \cdots$ denote the functions $x, e(x), e[e(x)]$, $e\{e[e(x)]\}, \cdots$ respectively. Furthermore let $F(x)$ denote an arbitrary matrix of single-valued analytic functions. A matrix $G(x)$ of single-valued analytic functions can then be found whose singular points fall at those of $F\left[e_{k}(x)\right]$ $(k=0,1,2, \cdots)$ and at the limit points $[c]$ of this set, and such that $G(x)$ is equivalent to $G[e(x)] F(x)$ save at these limit points.

Let us write down for reference the symbolic infinite product to the left

$$
\cdots F\left[e_{2}(x)\right] F\left[e_{1}(x)\right] F\left[e_{0}(x)\right] \text {. }
$$

The singular points of any factor $F\left[e_{n}(x)\right]$ not belonging to [c] are isolated singular points.

Consider now any such singular point $\bar{x}$ not a point of $[c]$. It is possible to take a positive integer $k$ so large that all the factors before the $k$ th in the above product are not singular at $\bar{x}$ nor in its neighborhood, since $\bar{x}$ is not a point of $[c]$.

We shall demand of a matrix $G(x)$ that it be equivalent to the last $k$ factors of the product

at every point $\bar{x}$.

$$
A\left[e_{k-1}(x)\right] \cdots A\left[e_{0}(x)\right]
$$

It is clear that if $k$ be increased $G(x)$ will still have the same property since the factors introduced are not singular at $\bar{x}$, and appear on the left of the previous factors.

The set of such points $\bar{x}$ is clearly numerable. In fact any set of isolated points is numerable.

According to $\$ 8$ a matrix $G(x)$ may be found which satisfies these relations of equivalence at the singular points $\bar{x}$ of $F\left[e_{m}(x)\right](m=0,1, \cdots)$ and which has no further singular points save the points of $[c]$.

This matrix $G(x)$ will have the desired property that $G(x)$ is equivalent to $G[e(x)] F(x)$ save at the points of $[c]$.

In proving this statement it is first to be observed that if $\bar{x}$ is a singular point of $F\left[e_{m}(x)\right]$, and if a value $\bar{y}$ exists such that $\bar{x}=e(\bar{y})$, then $\bar{y}$ is a singular point of $F\left[e_{m+1}(y)\right]$. For we have identically

$$
F\left[e_{m}(x)\right]=F\left[e_{m+1}(y)\right],
$$

where $x=e(y)$. Hence, if the matrix on the right is not singular at $\bar{y}$, the elements of the equal matrix on the left are analytic in $x$ at $\bar{x}$, since they can not be multiple-valued functions of $x$. Moreover, since as $x$ approaches $\bar{x}$, the variable $y$ approaches $\bar{y}$, the determinant of the right-hand side will not 
approach zero in this case. Thus $F\left[e_{m}(x)\right]$ would not be singular at $\bar{x}$, contrary to hypothesis.

The converse statement is obviously true, i. e., if $F\left[e_{m+1}(y)\right]$ is singular at $\bar{y}$ and if $\bar{x}=e(\bar{y})$, then $F\left[e_{m}(x)\right]$ is singular at $\bar{x}$.

Thus we derive the precise totality of singular points of successive factors of the symbolic infinite product from the singularities $\bar{x}_{0}$ of the first factor $F(x)$ by forming $e_{-1}\left(\bar{x}_{0}\right), e_{-2}\left(\bar{x}_{0}\right), \cdots$ where $e_{-1}(x), e_{-2}(x), \cdots$ denote the inverse functions to $e_{1}(x), e_{2}(x), \cdots$ respectively, which are of course in general multiple valued.

Consider now any point not belonging to [c].

If this point $\xi$ is not a singular point of some matrix $F\left[e_{m}(x)\right]$ then $G(x)$ is certainly not singular at $\xi$, as has been stated. Moreover $e(\xi)$ is not a singular point for $G(x)$, inasmuch as in the contrary case some $F\left[e_{m}(x)\right]$ is singular at $e(\xi)$ and thus the corresponding $F\left[e_{m+1}(x)\right]$ is singular at $\xi$. Consequently $G[e(x)$ ] is not singular at $\xi$. It follows that $G(x)$ is equivalent to $G[e(x)] F(x)$ at any such point $\xi$.

It remains to consider a point $\bar{x}$ for which some $F\left[e_{m}(x)\right]$ is singular. At such a point $G(x)$ has been chosen to be equivalent to a product

$$
F\left[e_{k}(x)\right] F\left[e_{k-1}(x)\right] \cdots F\left[e_{0}(x)\right],
$$

where $k$ has been taken so large as to include all the factors singular at $\bar{x}$. The point $e(\bar{x})$ will be a singular point for $F\left[e_{m-1}(x)\right]$ unless $m$ is zero. Putting aside this contingency for the moment, we see that $G(x)$ is equivalent to

$$
F\left[e_{k-1}(x)\right] F\left[e_{k-2}(x)\right] \cdots F\left[e_{0}(x)\right]
$$

at $e(\bar{x})$. Consequently $G[e(x)]$ is equivalent at $\bar{x}$ to

$$
F\left[e_{k}(x)\right] F\left[e_{k-1}(x)\right] \cdots F\left[e_{1}(x)\right] .
$$

Hence $G(. x)$ is equivalent to $G[e(x)] F(x)$ at such a point $\bar{x}$.

If, on the other hand, $m$ is zero then the integer $k$ may be taken zero. In this case $G(x)$ is equivalent to $F(x)$ at $\bar{x}$, while $G(x)$ is not singular at $e(\bar{x})$. In this case also $G(x)$ is equivalent to $G[e(x)] F(x)$ at $\bar{x}$.

The italicized statement made is now proved.

The usual existence theorems for linear difference and $q$-difference equations show that if $e(x)=x+1$ or $q x$ respectively, and if $F(x)$ is a polynomial matrix we may choose $G(x)$ equal to $G[e(x)] F(x)$.

HARVARD UNIVERSITY

March, 1916 